\title{
How to Effectively Reduce the Job Burnout of Front-line Financial Practitioners-Research on the Intermediary Effect of Work Engagement
}

\author{
Lin Guo ${ }^{1}$, Wenqi Wei ${ }^{1, *}$ and Long Cheng ${ }^{2}$ \\ ${ }^{1}$ School of Government, Beijing Normal University, Beijing, 100875, China \\ ${ }^{2}$ Strategic Talent Research Centre, Beijing Normal University, Beijing, 100875, China \\ *Corresponding author. Email: 201821260027@mail.bnu.edu.cn
}

\begin{abstract}
This paper analyzes the job burnout status of front-line financial practitioners and the direct impact of job crafting on job burnout, and examines the mediating role of job engagement between job crafting and job burnout. A questionnaire survey method was used to collect data for multi-level regression analysis. The results of the study found that front-desk staff, male employees, and young employees with relatively short working years who directly face customers are groups with high job burnout; job crafting can effectively reduce job burnout. Job burnout of front-line financial practitioners; job engagement has a partial mediating effect between job crafting and job burnout, that is, job crafting can reduce job burnout by increasing job engagement. Financial industry managers should make full use of the impact of job crafting, and use performance appraisal reforms and organizational culture construction to fully guide front-line financial practitioners to change all aspects of their work and reduce job burnout effectively.

Keywords: Job crafting; Job burnout; Engagement; Mediating effect
\end{abstract}

\section{INTRODUCTION}

In recent years, with the rapid development of the Internet, information technology and other fields, on the one hand, it has provided convenient conditions for the extended development of the financial industry. On the other hand, it has also brought about an impact on the traditional financial industry, and the job burnout of front-line financial practitioners is getting more and more attention from the society, and it is more common for some financial professionals to leave. Therefore, as managers in the financial industry, leaders need to transform their thinking from management practices [1], and explore effective ways and methods to reduce the job burnout of front-line financial practitioners at the grassroots level, and ultimately achieve the goal of reducing the turnover rate of front-line financial practitioners.

Under this new situation, financial industry managers need to further stimulate the enthusiasm of financial practitioners and reduce their job burnout through management measures such as job design or job redesign. Front-line financial practitioners also need to explore and discover the meaning and value of work actively in their own jobs, in order to make them more engage in and accept their work [2], which will greatly reduce their own job burnout. So how to guide them to start proactively from themselves and make appropriate changes to all aspects involved in the work from the bottomup, in other words, how to guide financial practitioners to carry out reasonable, efficient job crafting and reduce the occurrence of resignation imagination has become a problem that managers in the financial industry need to solve urgently. Therefore, this research analyses the job burnout status of front-line financial practitioners at the grassroots level, the direct impact of job crafting on job burnout and examines the intermediary effect of job engagement between job crafting and job burnout. The path and method to reduce the job burnout of the front-line financial practitioners at the grassroots level will have important theoretical and practical value for stabilizing the backbone of front-line financial talents and improving the development level of the national financial industry.

\section{OBJECTS AND METHODS}

\subsection{Research Object}

Distributed questionnaires to front-line employees of basic financial units such as bank branches and branches, stock exchange branches, insurance company branches, etc. in some provinces and cities such as Beijing, Chongqing, etc., and stated that all contents will be kept strictly confidential and used for academic research only. In the end, 267 questionnaires were distributed, unqualified questionnaires were deleted, and 251 valid questionnaires were finally retained. The effective recovery rate was $94.1 \%$. Among the 251 valid questionnaires, the front desk positions accounted for $34.6 \%$, the financial settlement category accounted for $40.1 \%$, and the back-office customer docking category accounted for $25.3 \%$; unmarried $47.2 \%$, married $57.8 \%$; male 
$30.6 \%$, female $69.4 \%$; 30 years old $43.1 \%$ and below $43.1 \%$, $33.6 \% 31-40$ years old, $23.3 \% 41$ years old and above; $39.2 \%$ of working years 5 years and below, 30.2\% 6-10 years, and $31.6 \% 11$ years and above.

\subsection{Research Tools}

Adopting a well-recognized and more authoritative scale, and crafting the work using a scale developed by Tims and Bakker [4]. The scale contains four dimensions and a total of 21 questions. The internal consistency coefficient (Cronbach $\alpha$ ) of the questionnaire is 0.791 , indicating that the questionnaire It has good reliability; the work engagement is based on a scale developed by Schaufeli et al. [5] that includes 9 questions in three dimensions: vitality, dedication, and focus. The internal consistency coefficient (Cronbach $\alpha$ ) of the questionnaire is 0.886 , indicating that the questionnaire has Good reliability; job burnout adopts a scale developed by Konovsky MA and Cropanzano R[6] with a dimension containing 3 questions. The internal consistency coefficient of the questionnaire (Cronbach $\alpha$ ) is 0.803 , which shows that the questionnaire has good reliability.

In addition, in order to better analyze the job burnout of financial practitioners in different situations, the questionnaire added the personal information items of the person's gender, marriage, age, working years, and job position. All questionnaire topics and personal information use Li The Kurt five-point scale method, that is, "1" means strongly disagree, "2" means disagree, "3" means uncertain, "4" means agree, and "5" means very agree.

\subsection{Statistical Analysis Methods and Tools}

After careful screening of the collected data, bad data was deleted, and SPSS18.0 was used for descriptive statistical analysis and multi-level linear regression analysis.

\section{RESEARCH RESULTS AND ANALYSES}

\subsection{Job Burnout of Financial Employees in Financial Institutions}

This study found that there are obvious differences in job burnout among financial practitioners in different situations, which are mainly manifested in three aspects: First, the job burnout of financial practitioners in different jobs is highest in front desk staff, followed by financial settlement and other positions. , The back-end customer docking category is once again; second, the job burnout of male financial practitioners is significantly higher than that of females; third, the job burnout of young financial practitioners with a shorter working life of 5 years or less is significantly higher than that of 6-10 years, 11 Financial practitioners of years and above.

\subsection{The Direct Impact of Job Crafting on Job Burnout}

Excluding the influence of relevant individual variables on the multi-level regression analysis, five variables including gender, marriage, age, working years, and job position are used as control variables (model M1), and the mediation effect is tested on this basis. Then take job crafting as the independent variable and job burnout as the dependent variable to obtain the relationship between the independent variable and the dependent variable (model M2). The results in Table 1 show that job crafting is significantly negatively correlated with job burnout $(-0.365, \mathrm{p}<0.001)$, that is, when the job crafting of financial practitioners increases, their job burnout level will be significantly reduced.

\subsection{The Mediating Role of Job Engagement Between Job Crafting and Job Burnout}

Taking job crafting as the independent variable and job burnout as the dependent variable, the job engagement degree is added as an intermediary variable to the model to test the mediating effect of job engagement degree (model M3). The results in Table 1 show that when job engagement is added to the model, job crafting still has a significant negative correlation with job burnout, but the absolute value of the correlation coefficient becomes smaller $(-0.146, p<0.001)$, and work Engagement degree and job burnout are also significantly negatively correlated $(-0.303, \mathrm{p}<0.001)$, that is, job engagement degree has a partial mediating effect between job crafting and job burnout.

Table 1. The mediating role of job engagement

\begin{tabular}{|c|c|c|c|c|}
\hline IV & \multicolumn{2}{|c|}{ Burnout } & \multicolumn{2}{|c|}{ Engagement } \\
\hline Model & M1 & M2 & M3 & M4 \\
\hline \multicolumn{5}{|l|}{$1^{\text {st }}: C V$} \\
\hline Gen & -0.016 & -0.023 & 0.004 & 0.027 \\
\hline Year & -0.044 & -0.103 & -0.86 & $-0.172^{* *}$ \\
\hline Age & -0.040 & -0.049 & -0.011 & 0.080 \\
\hline Position & 0.078 & 0.044 & 0.019 & -0.033 \\
\hline Marriage & -0.003 & -0.022 & -0.037 & 0.015 \\
\hline \multicolumn{5}{|l|}{$2^{\text {ndd }}: I V$} \\
\hline JC & & $-0.365^{* * *}$ & $-0.146^{* * *}$ & $0.222^{* * *}$ \\
\hline \multicolumn{5}{|l|}{$3^{\text {rd }}: M V$} \\
\hline Engage & & & $-0.303^{* * *}$ & \\
\hline$R^{2}$ & 0.026 & 0.157 & 0.238 & 0.255 \\
\hline$\Delta R^{2}$ & $0.026^{*}$ & $0.131^{* * *}$ & $0.081^{* *}$ & $0.229^{* * *}$ \\
\hline Note: & $1, *$ & 05 & $01, * * *$ & 0.001 \\
\hline
\end{tabular}




\section{RESEARCH DISCUSSION}

\subsection{Analysis of Job Burnout of Financial Practitioners in Different Situations}

In view of the job burnout situation of front-line financial practitioners shown by the research results, it is found that people in front-end positions, male financial practitioners, and young financial practitioners with relatively short working years are the groups with higher job burnout. In interviews with this part of the staff, it was found that this was due to the fact that the front desk staff faced the most trivial but most concerned basic financial service business of the masses, and had high requirements for staff patience, business knowledge mastery, and emotional self-regulation ability. As a result, some front-office employees feel greater work pressure; male financial practitioners bear more family economic responsibilities and value their personal career development more. Therefore, once the salary package and career promotion are not due, they are likely to have Work frustration leads to a willingness to resign; young financial practitioners have not yet formed a clear career development plan because of their short working years. Once they face a huge gap between their actual work and their ideals in a financial unit, they are also likely to have a tendency to resign. It can be seen that these three types of financial practitioners are the groups that financial unit managers need to pay most attention to, and they need to effectively reduce their job burnout from the perspective of job crafting and maintain the talent turnover rate in a reasonable state [7].

\subsection{The Mitigation Effect of Job Crafting on Job Burnout}

The results of the study show that job crafting has a negative correlation with financial practitioners' job burnout $(-0.404$, $\mathrm{p}<0.001)$, that is, the higher the level of job crafting of financial practitioners, the less likely they will be to resign. Therefore, financial unit leaders can guide financial practitioners to actively change their behavior and performance at work through a variety of ways to make their work more meaningful; actively change the content and methods of interpersonal interaction at work to improve their interpersonal relationship Relationship, create a good working atmosphere; look at your work from another angle to gain different understanding and feelings, feel the value that this sacred work brings to the convenience of the majority of residents, so as to generate a desire to devote yourself to work, and finally Reduce the willingness to resign [8] and stabilize the team of financial practitioners.

\subsection{The Mediating Role of Work Engagement}

The research results also show that job remodeling can reduce job burnout by increasing job engagement. Therefore, if leaders of financial units want to guide financial practitioners to remodel jobs to reduce job burnout, it is also good to start with job engagement. way. Financial unit managers should actively integrate into the frontline financial practitioners, listen to their true feelings about the current work situation, and focus on maintaining the finances on the most prominent issues such as labor relations disputes, unclear division of work tasks, unfair and opaque assessment systems, etc. The benefits of practitioners[9] enable them to be proactive and willing to invest more in front-line financial work, thereby achieving the purpose of reducing the willingness to leave and stabilizing the team of financial practitioners.

\section{ACKNOWLEDGMENT}

This study verifies the relationship between job remodeling and financial employees' job burnout in financial institutions, and explores the mediating role of job engagement between job remodeling and job burnout. The results prove that job remodeling can effectively reduce job burnout of financial practitioners in financial institutions; in addition to job remodeling can directly alleviate job burnout, job remodeling can also reduce job burnout by increasing work engagement. Financial unit managers should make full use of the impact of work remodeling, and use performance appraisal reforms [9], organizational culture construction[10] and other ways to fully guide financial practitioners in financial units to reshape tasks, relationships, and perceptions of work, so as to achieve effective The purpose of reducing job burnout is to finally achieve the stability of the financial staff of financial institutions and improve the development level of China's financial industry.

\section{REFERENCES}

[1] Long Cheng, Haibo Yu. How transformational and transactional leaders promote organizational learningBased on the complete mediating role of organizational culture[J]. Journal of Shandong University of Finance and Economics, 2018,30(06):99-109. (In Chinese)

[2] Wrzesniewski, A., Dutton, J. E.. Crafting a job: Revisioning employees as active crafters of their work[J]. Academy of Management Review, 2001, 26(2): 179-201.

[3] Ruiling Hu. Research on the relationship between job value orientation, job remodeling and job satisfaction [D]. Chongqing: Chongqing Technology and Business University, 2016.

[4] Bakker, A.B., Tims, M. \&Derks, D. Proactive personality and job performance: the role of job crafting and work engagement[J]. Human Relations, 2012, 65(10): 1359-1378. (In Chinese)

[5] Schaufeli, Salanova, Gonzálezromá, Bakker. The measurement of engagement and burnout: A two sample 
confirmatory factor analytic approach[J]. Journal of Happiness Studies, 2002, 3(1):71-92.

[6] Konovsky MA, Cropanzano R. Perceived fairness of employee drug testing as a predictor of employee attitudes and job performance[J]. Journal of Applied Psychology, 1991, 76 (5): 698-707.

[7] Long Cheng, Haibo Yu, Zhang Lu, An Ran. How does the territorial behavior of scientific and technological talents affect their job burnout: An intermediary model in which marital status and psychological ownership are regulated[J]. Science and Technology Progress and Policy, 2019, 36(13) :131-137. (In Chinese)
[8] Long Cheng, Haibo Yu. Research on the mobility policy of science and technology talents in universities from the perspective of supply side[J]. Science and Technology of Chinese Universities, 2018(12): 10-13. (In Chinese)

[9] Zhenbang Fang. Performance Management [M]. Beijing: Renmin University of China Press, 2012. (In Chinese)

[10] Long Cheng, Haibo Yu. How transformational and transactional leaders promote organizational learning Based on the complete mediation of organizational culture[J]. Journal of Shandong University of Finance and Economics, 2018, 30(06): 99-10. (In Chinese) 